\title{
A Secreted Protein in the Wnt Signaling Pathway-R-Spondin3: A Mini-Review
}

\author{
Zhilong Chen ${ }^{1,2}$, Jian Zhang ${ }^{1}$, Qing Yang ${ }^{2 *}$ and Xiujun Fan ${ }^{1 *}$ \\ ${ }^{1}$ Shenzhen Institutes of Advanced Technology, China \\ ${ }^{2}$ Department of Clinic Veterinary Medicine, Human Agricultural University, China
}

*Corresponding author: Xiujun Fan, Laboratory for Reproductive Healthy, Shenzhen Institutes of Advanced Technology, Chinese Academy of Sciences, 1068 Xueyuan Avenue, Shenzhen University Town, Shenzhen, China

Qing Yang, Department of Clinic Veterinary Medicine, College of Veterinary Medicine, Hunan Agricultural University, Nongda Road, Furong district, Changsha, China

Submission: March 16, 2018; Published: May 22, 2018

\begin{abstract}
R-spondins (Roof plate-specific Spondins, RSPOs) are secreted proteins, which act as activators of Wnt/ $\beta$-catenin signaling and play roles in cell proliferation and differentiation, embryonic development, vasculogenesis as well as many human diseases. R-spondin3 (RSPO3) is a member of RSPOs family, containing two adjacent cysteine-rich furin-like domains, which act as an agonist in Wnt signaling. In this mini-review, we discuss and classify recent progress in understanding the protein functions of RSPO3 in embryonic development, vasculogenesis, and its role in cancers.
\end{abstract}

Keywords: RSPO3; Wnt signaling; Embryonic development; Vasculogenesis; Cancers

\section{Introduction}

The Wnt signaling pathway is one of the key signaling pathways which controls cell proliferation and differentiation, muscle development, bone formation, morphogenesis during embryo genesis and in adults [1,2]. The R-spondins (Roof plate-specific Spondins, RSPOs) is a recently reported protein family including four secreted proteins (RSP01-4). RSPOs contain two furin-like cysteine-rich domains, a thrombospondin Type 1 repeat and a C-terminal region enriched with basic charged amino acids [3]. Leucine-rich repeat-containing G-protein coupled receptors (LGR 4-6) and ZNRF3/RNF43 are considered obligate high-affinity receptors of RSPOs [4]. They can potently enhance Wnt signals by amplifying target cell sensitivity to Wnt ligands through increasing Wnt receptor levels [5,6] (Figure 1A). RSPO3 is a member of the RSPO family, in vertebrates, which can activate the Wnt signaling and play important roles in embryonic patterning, vasculature remodeling and tumorigenesis [7-10].

RSPOs-mediated Wnt signaling activation. The $\beta$-catenin signaling is initiated by association of Wnt with Fizzled and LRP5/6 receptors, it subsequently activates Dishevelled to recruit the Axin complex (GSK-3 $\beta$, CK1 $\gamma$, Axin, APC) to the receptor. When RSPOs bind to LGR4/5/6 and RNF43/ZNRF3 receptors, the complex is internalized by endocytosis and induced ubiquitination and degradation of RNF43/ZNRF3 to stabilize Wnt receptors and enhance Wnt response, $\beta$-catenin becomes stabilized and enters the nucleus. B, Domains of RSP03. LGR4/5/6,Leucine-rich repeat- containing G-protein coupled receptors 4/5/6; RNF43, Ring finger protein 43; ZNRF3, Zinc and ring finger 3; LRP5/6, Low-density lipoprotein receptor-related protein 5/6; HSPG, Heparan sulphate proteo glycans; DVL, Dishevelled; GSK-3 $\beta$, Glycogen synthase kinase-3 $\beta$; CK1 $\gamma$, Casein kinase 1; TCF, T-cell factor; FU, Furin-like domains; N-gly, N-glycosylation; TSP-1, Thrombospondin-1; BR, Basic residues.

\section{RSPO3 and its Roles}

RSP03, also known as cysteine-rich and single thrombospondin domain containing- 1 , is a31 $\mathrm{kDa}$ secreted protein, which shares about $40 \%$ amino acid (aa) identity with the other three RSPO family members $[11,12]$. Each RSPO family member has a distinct expression pattern to potentiate Wnt/ $\beta$-catenin signaling. RSPO3 contains two adjacent cysteine-rich furin-like domains (aa 35 135) with one potential N-glycosylation site (aa 36), followed by a thrombospondin (TSP-1) motif (aa 147 207) and a region rich in basic residues (aa 211269 ) (Figure 1B). Only the furin-like domains are needed for $\beta$-catenin stabilization [12]. Within aa 21209 , human RSPO3 shares 93\%, 92\%, 97\%, 96\% and 92\% aa identity with mouse, rat, equine, bovine and canine RSPO3, respectively [13], so they have similar function in vertebrate development.

It is reported that RSPO3 is highly expressed in vascularized tissues such as coronary stems, theallantois and the primitive streak and controls vascular morphogenesis in zebrafish, Xenopus, mouse and human $[14,15]$. Embryonic lethality in Rspo3-deficient 
mice is caused due to vascular defects in placenta $[7,16]$. Inducible deletion of endothelial RSPO3 resulted in perturbed developmental and tumor vascular remodeling with reduced micro vessel density [8]. Tissue-specific ablation of RSPO3 in the heart with the Islet1Cre line leads to defective secondary heart field development [17]. RSPO3 is a crucial regulator of coronary artery formation in the developing heart [18], but also shown to activate the non-canonical
Wnt/calcium pathway in the endothelial compartment of the postnatal retina and lungs to promote blood vessel maintenance [8]. Moreover, RSPO3 can protect tissues against mesenteric ischemia/reperfusion by tightening endothelial cell junctions and improving vascular integrity [19]. Thus, RSPO3 plays an important role in angiogenesis and vessel sprouting.

A

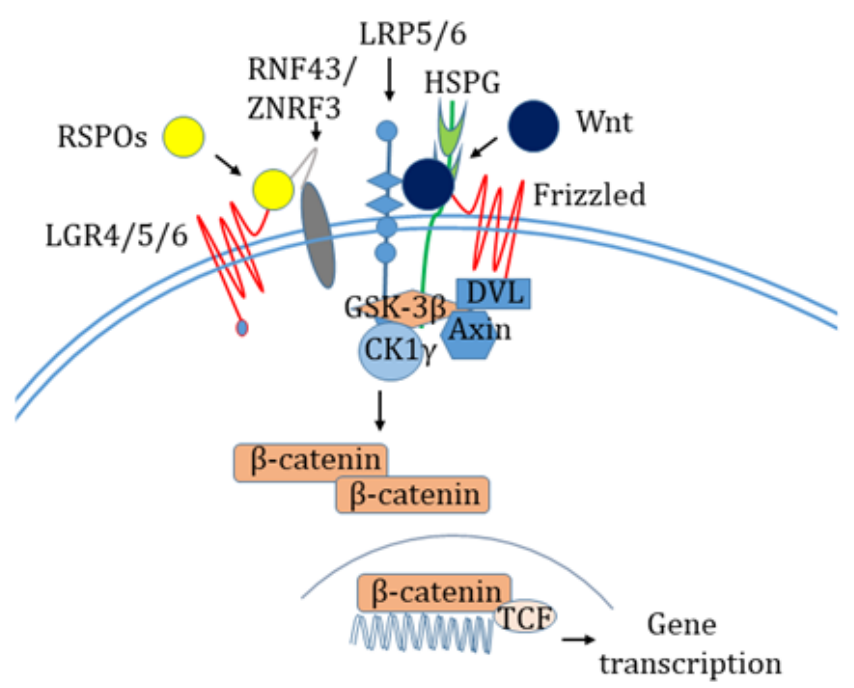

B

Figure 1: Overview of RSPOs activating Wnt signaling and RSPO3 domains.

RSPO3 can induce and synergize with Wnt ligands to maintain homeostasis in normal tissues by Wnt pathway. It has been shown that Wnt signaling is markedly activated in several cancers [20]. Recurrent RSPO3 translocation was identified in $8.0 \%$ of colon cancer. There are two configurations of the fusion transcripts, known as PTPRK (exon1)-RSPO3 (exon2) and PTPRK (exon7)RSPO3 (exon2) [21,22]. Indeed, high frequencies of PTPRK-RSPO3 fusions (31\%) and RNF43 mutations (24\%) have been reported in colorectal traditional serrated adenomas [23]. Interestingly, RSPO3 fusions are found in prostate cancers, lung cancer and Schwannoma [24,25], and it is identified as sites of integration for mouse mammary tumor virus induced mammary tumors [26,27]. Furthermore, involvement of RSPO3 in growth of pre-existing tumors was recently shown in RSPO3-fusion-positive xenograft models, where anti-RSPO3 treatment inhibited the tumor growth $[22,23]$. RSPO3 is an oncogenic driver, rapidly causing intestinal cancer and extensive crypt hyperplasia, concomitantly stimulating stem cells and supportive niche cells [10]. Therefore, identification of RSP03 translocations in cancer provides an attractive target for Wnt pathway inhibition using antibody approaches and an intuitive predictive biomarker for RSPO3 antibodies. These might thus serve as predicative biomarkers to identify Wnt-dependent tumors.

\section{Conclusion}

In summary, RSPO3 not only contributes to the embryonic development, but also promotes angiogenesis and vessel sprouting. It also has been recognized as an oncogene to actively drive tumor genesis. Therefore, RSPO3 may be as a useful candidate target for underlying the etiology of some human diseases even in cancers.

\section{Acknowledgement}

The work is supported by NSFC $(31572591 ; 31272630)$ to Qing Yang, NSFC (81771611), the "Organ Reconstruction and Manufacturing", Strategic Priority Research Program of the Chinese Academy of Sciences, Grant No. XDA16020702 to Jian Zhang, 2016A030313178, NSFC (81571445; 81771617) to Xiujun Fan.

\section{References}

1. Niehrs C (2012) The complex world of WNT receptor signalling. Nat Rev Mol Cell Biol 13(12): 767-779. 
2. Yang Y (2012) Wnt signaling in development and disease. Cell Biosci 2(1): 14.

3. De Lau WB, Snel B, Clevers HC (2012) The R-spondin protein family. Genome Biol 13(3): 242.

4. Glinka A, Dolde C, Kirsch N, Huang YL, Kazanskaya O, et al. (2011) LGR4 and LGR5 are R-spondin receptors mediating Wnt/ $\beta$-catenin and Wnt/ PCP signalling. EMBO Rep 12(10): 1055-1061.

5. Liu H, Zhou Y, Tan F, Wang Y, Pei H (2017) Expression of regulatory factor R-spondin family in Wnt signaling pathway in colorectal cancer and its clinical significance. Zhong Nan Da Xue Xue Bao Yi Xue Ban 42(5): 501506.

6. Yan KS, Janda CY, Chang Y, Zheng GXY, Larkin KA, et al. (2017) Nonequivalence of Wnt and R-spondin ligands during Lgr5(+) intestinal stem-cell self-renewal. Nature 545(7653): 238-242.

7. Aoki M, Mieda M, Ikeda T, Hamada Y, Nakamura H, et al. (2007) R-spondin3 is required for mouse placental development. Dev Biol 301(1): 218-226

8. Scholz B, Korn C, Wojtarowicz J, Mogler C, Augustin I, et al. (2016) Endothelial RSPO3 controls vascular stability and pruning through noncanonical WNT/Ca (2+)/NFAT signaling. Dev Cell 36(1): 79-93.

9. Fischer MM, Yeung VP, Cattaruzza F, Hussein R, Yen WC, et al. (2017) RSPO3 antagonism inhibits growth and tumorigenicity in colorectal tumors harboring common Wnt pathway mutations. Sci Rep 7(1): 15270.

10. Hilkens J, Timmer NC, Boer M, Ikink GJ, Schewe M, et al. (2017) RSPO3 expands intestinal stem cell and niche compartments and drives tumorigenesis. Gut 66(6): 1095-1105.

11. Carmon KS, Gong X, Lin Q, Thomas A, Liu Q (2011) R-spondins function as ligands of the orphan receptors LGR4 and LGR5 to regulate Wnt/betacatenin signaling. Proc Natl Acad Sci U S A 108(28): 11452-11457.

12. Kim KA, Wagle M, Tran K, Zhan X, Dixon MA, et al. (2008) R-Spondin family members regulate the Wnt pathway by a common mechanism. Mol Biol Cell 19(6): 2588-2596.

13. Kim KA, Zhao J, Andarmani S, Kakitani M, Oshima T, et al. (2006) R-Spondin proteins: a novel link to beta-catenin activation. Cell Cycle 5(1): $23-26$

14. Da Silva F, Rocha AS, Motamedi FJ, Massa F, Basboga C, et al. (2017) Coronary artery formation is driven by localized expression of R-spondin3. Cell Reports 20(8): 1745-1754.
15. Nam JS, Turcotte TJ, Yoon JK (2007) Dynamic expression of R-spondin family genes in mouse development. Gene Expr Patterns 7(3): 306-312.

16. Kazanskaya O, Ohkawara B, Heroult M, Wu W, Maltry N, et al. (2008) The Wnt signaling regulator R-spondin 3 promotes angioblast and vascular development. Development 135(22): 3655-3664.

17. Cambier L, Plate M, Sucov HM, Pashmforoush M (2014) Nkx2-5 regulates cardiac growth through modulation of Wnt signaling by R-spondin3. Development 141(15): 2959-2971.

18. Fujiwara M, Kato S, Niwa Y, Suzuki T, Tsuchiya M, et al. (2016) C-mannosylation of R-spondin3 regulates its secretion and activity of Wnt/ß-catenin signaling in cells. FEBS Lett 590(16): 2639-2649.

19. Kannan L, Kis-Toth K, Yoshiya K, Thai TH, Sehrawat S, et al. (2013) R-spondin3 prevents mesenteric ischemia/reperfusion induced tissue damage by tightening endothelium and preventing vascular leakage. Proc Natl Acad Sci USA 110(35): 14348-14353.

20. Anastas JN, Moon RT (2013) WNT signalling pathways as therapeutic targets in cancer. Nat Rev Cancer 13(1): 11-26.

21. Seshagiri S, Stawiski EW, Durinck S, Modrusan Z, Storm EE, et al. (2012) Recurrent R-spondin fusions in colon cancer. Nature 488(7413): 660664.

22. Storm EE, Durinck S, Sousa MF, Tremayne J, Kljavin N, et al. (2016) Targeting PTPRK-RSPO3 colon tumours promotes differentiation and loss of stem-cell function. Nature 529(7584): 97-100.

23. Sekine S, Yamashita S, Tanabe T, Hashimoto T, Yoshida H, et al. (2016) Frequent PTPRK-RSPO3 fusions and RNF43 mutations in colorectal traditional serrated adenoma. J Pathol 239(2): 133-138.

24. Robinson D, Van Allen EM, Wu YM, Schultz N, Lonigro RJ, et al. (2015) Integrative clinical genomics of advanced prostate cancer. Cell 161(5): $1215-1228$.

25. Watson AL, Rahrmann EP, Moriarity BS, Choi K, Conboy CB, et al. (2013) Canonical Wnt/beta-catenin signaling drives human schwann cell transformation, progression, and tumor maintenance. Cancer Discov 3(6): 674-689.

26. Hao HX, Jiang X, Cong F (2016) Control of Wnt receptor turnover by R-spondin-ZNRF3/RNF43 signaling module and its dysregulation in cancer. Cancers (Basel) 8(6).

27. Callahan R, Mudunur U, Bargo S, Raafat A, McCurdy D, et al. (2012) Genes affected by mouse mammary tumor virus (MMTV) proviral insertions in mouse mammary tumors are deregulated or mutated in primary human mammary tumors. Oncotarget 3(11): 1320-1334.
Creative Commons Attribution 4.0 nternational License

For possible submissions Click Here

\section{Submit Article}

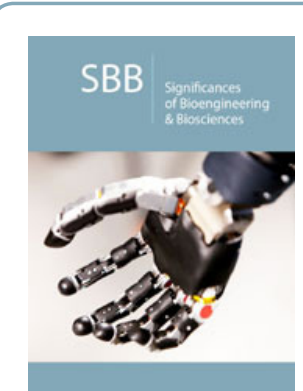

Significances of Bioengineering \& Biosciences

\section{Benefits of Publishing with us}

- High-level peer review and editorial services

- Freely accessible online immediately upon publication

- Authors retain the copyright to their work

- Licensing it under a Creative Commons license

- Visibility through different online platforms 